\title{
Cerebral Infarction in Immune Thrombotic Thrombocytopenic Purpura Is Associated with Old Age, Hypertension, Smoking, and Anti-ADAMTS13 lg, But Not with Mortality
}

\author{
Raima Memon ${ }^{1}$ Jingrui Sui ${ }^{1}$ Chen Lin $^{2} \quad$ X. Long Zheng ${ }^{1,3}$ \\ ${ }^{1}$ Division of Laboratory Medicine, Department of Pathology, \\ The University of Alabama at Birmingham, Birmingham, \\ Alabama, United States \\ 2 Department of Neurology, The University of Alabama at \\ Birmingham, Birmingham, Alabama, United States \\ Address for correspondence X. Long Zheng, MD, PhD, Department of \\ Pathology and Laboratory Medicine, University of Kansas Medical \\ Center, 3901 Rainbow Blvd, 5016 Delp, Kansas City, KS 66160, \\ United States \\ (e-mail: xzheng2@kumc.edu; longzheng01@gmail.com).
}

${ }^{3}$ Department of Pathology and Laboratory Medicine, University of Kansas Medical Center, Kansas City, Kansas, United States

TH Open 2021;5:e1-e7.

\begin{abstract}
Keywords

- thrombotic thrombocytopenic purpura

- inflammatory mediators

- ADAMS/ADAMTS13

- von Willebrand factor

- cerebrovascular disease

- autoimmune diseases

- autoantibodies

Background Neurological involvement is common in patients with immune thrombotic thrombocytopenic purpura (iTTP), but the frequency, risk factors, and outcomes of these with imaging-confirmed stroke in ITTP are not known.

Methods We selected 66 out of 109 iTTP patients with neurological signs and symptoms and reviewed their CT/MRI (computed tomography/magnetic resonance imaging) findings for the evidence of stroke and other clinical information in Alabama TTP Registry.

Results Of these, 52 (78.8\%) had their CT/MRI done on admission in whom 22 (42.3\%) were positive for multiple acute or chronic infarcts. The patients with image-confirmed ischemic stroke were older, and appeared to be associated with a history of hypertension and smoking. Additionally, patients with imaging-confirmed stroke showed higher plasma concentrations of anti-ADAMTS13 $\mathrm{lgG}$ than those without stroke. More interestingly, there was no statistically significant difference in the rate of exacerbation and 60-day mortality between those with and without stroke.

Conclusion Ischemic cerebral infarcts are common findings in brain imaging studies of patients with acute iTTP; old age, chronic hypertension, and smoking, as well as high plasma concentrations of anti-ADAMTS13 IgG may be the potential risk factors for cerebral infarction in these patients. The presence of image-confirmed ischemic stroke, however, does not predict exacerbation and 60-day mortality, although the long-term effect of such ischemic brain damage on cognitive function and quality of life remains to be determined.
\end{abstract}

received

November 17, 2020 accepted after revision November 30, 2020
DOI https://doi.org/

$10.1055 / \mathrm{s}-0040-1722610$. ISSN 2512-9465. (c) 2021. The Author(s).

This is an open access article published by Thieme under the terms of the Creative Commons Attribution License, permitting unrestricted use, distribution, and reproduction so long as the original work is properly cited. (https://creativecommons.org/licenses/by/4.0/)

Georg Thieme Verlag KG, Rüdigerstraße 14, 70469 Stuttgart, Germany 


\section{Introduction}

Immune-mediated thrombotic thrombocytopenia purpura (iTTP) is a rare but potentially fatal blood disorder. It is characterized clinically by severe thrombocytopenia (platelet count, usually less than $30 \times 10^{9} / \mathrm{L}$ ), microangiopathic hemolytic anemia (e.g., low hematocrit and hemoglobin, low haptoglobin, elevated lactate dehydrogenase (LDH), and presence of schistocytes, etc.), and organ injury. ${ }^{1,2}$ The incidence of iTTP ranges from two to six cases per million residents, ${ }^{3,4}$ but may be much higher in the African American population. ${ }^{4}$ The iTTP patients may present with a thrombotic event, either initially or during the course of treatment, including ischemic cerebral infarction (i.e., stroke), ${ }^{5-7}$ and acute myocardial infarction (MI). ${ }^{8-10}$ The clinical presentation may range from mild nonspecific symptoms, including weakness, fatigue, headache, nausea and vomiting to stroke, MI and acute renal failure, and death. ${ }^{11,12}$ The classic pentad with fever, thrombocytopenia, hemolytic anemia, neurological signs, and renal failure occurs in less than $10 \%$ of iTTP patients. ${ }^{13,14}$ Thus, the presence of thrombocytopenia and microangiopathic hemolytic anemia is sufficient for the presumptive diagnosis of TTP. ${ }^{15-17}$

To confirm the diagnosis of iTTP, plasma ADAMTS13 activity and inhibitors or anti-ADAMTS13 IgGs must be performed in a timely manner. ${ }^{18}$ ADAMTS13 is a plasma metalloprotease that cleaves von Willebrand factor (VWF), thus regulating normal hemostasis. ${ }^{19-21}$ If plasma ADAMTS13 is less than $10 \mathrm{U} / \mathrm{dL}$ (or $10 \%$ of normal), TTP diagnosis is confirmed; if plasma ADAMTS13 is greater than $20 \mathrm{U} / \mathrm{dL}$ (or $20 \%$ of normal), the TTP is essentially ruled out. If plasma ADAMTS13 activity is between 10 and $20 \mathrm{U} / \mathrm{dL}$ (or $10-20 \%$ of normal), clinical judgment is required to interpret this result. ${ }^{18}$ Most adult TTP patients (greater than 95\%) are caused by IgG type autoantibodies against ADAMTS13. ${ }^{4,22}$ A positive inhibitor test $(>0.4 \mathrm{U} / \mathrm{mL})$ or an increased level of anti-ADAMTS13 $\mathrm{IgG}$ ( $>15 \mathrm{U} / \mathrm{mL}$ ) is confirmatory for iTTP. ${ }^{4,15-17}$ However, a negative inhibitor or antibody test does not automatically rule out the diagnosis of iTTP. Further follow-up tests and the observation of response to therapeutic plasma exchange (TPE) may provide additional information for the final diagnosis of iTTP.

Neurological signs and symptoms are common as the initial complaints in many patients with iTTP. ${ }^{11,12,23}$ This may be resulted from the formation of microvascular thrombosis in small arteries and capillaries in the central nervous system, leading to transient (known as reversible encephalopathy ${ }^{24,25}$ or permanent ischemic brain damage. ${ }^{5-7}$ These thrombi in large or small vessels in the brain may cause these neurological signs and symptoms ranging from headache, forgetfulness, numbness, and weakness of the extremities, and confusion to more severe forms such as seizure, stroke, coma, and death. Therefore, early recognition and prompt treatments such as TPE, ${ }^{12,23,26}$ steroids, and caplacizumab (an anti-VWF nanobody) ${ }^{27-29}$ are important as these microthrombi can lead to permanent brain damage leading to high morbidity and mortality. Rituximab (an anti-CD20 monoclonal antibody) helps suppress antibody production, thus reducing relapses in these patients. ${ }^{30-37}$

Present study aims to determine the frequency and risk factors of ischemic stroke in patients with iTTP and the association of such an ischemic stroke with the exacerbation and 60-day mortality rate in these patients. The results of this study may help stratify patients for more aggressive treatment strategy, thus reducing long-term morbidity.

\section{Methods}

Patients: The study was approved by the Institutional Review Board (IRB) of the University of Alabama at Birmingham, Alabama. The patient cohort from April 2006 and December 2019 was included for the study. All the patients were referred to us for treatment with TPE. Of 109 episodes, 66 unique patients with confirmed iTTP and presenting neurological symptoms at the time of the admission were identified for the study. The neurological symptoms range from mild complaints of headache, dizziness to severe complaints of slurring of speech, forgetfulness, visual changes, numbness, and weakness of the extremities or coma, and seizures.

Patient's clinical information was extracted from electronic medical records for patient demographics (e.g., age, sex, race, blood group) and presence of any comorbidities in these patients (e.g., hypertension, diabetes, history or current smoker, cancer, HIV, other autoimmune diseases, etc.). The data for these patients was further searched for management of these patients when presenting with these neurological symptoms and if neuroimaging studies (either computed tomography, CT or magnetic resonance imaging, MRI) were performed at the time of presentation for a suspected stroke or transient ischemic attack. For the patients who had CT/MRI done, results of the imaging were reviewed by a vascular neurologist to identify the presence or absence of confirmed stroke.

Sample collection and laboratory testing: All patients with confirmed iTTP included in this study had blood samples collected at the time of presentation or prior to initial TPE. Whole blood was drawn from an apheresis catheter and anticoagulated with $0.32 \%$ sodium citrate and centrifuged at $1,500 \times \mathrm{g}$ for 15 minutes. Plasma was separated from cellular components within 2 hours of collection and stored in aliquots at $-80^{\circ} \mathrm{C}$ until testing.

The laboratory parameters, including complete blood count, white blood count, hematocrit, platelet count, creatinine, prothrombin time (PT), partial prothrombin time, fibrinogen, D-dimer, LDH, and troponin-I were all performed routinely for patient care and the data were extracted from electronic medical record or the Alabama Registry database.

ADAMTS13 activity and inhibitor titers were determined in the reference laboratory (Versiti, Milwaukee, Wisconsin).

Anti-ADAMTS13 IgG was determined using a commercial ELISA kit (DiaPharma, West Chester, Ohio) according to the manufacturer's protocol. ${ }^{38}$

Plasma histone-DNA complexes (Millipore Sigma), cell-free DNA (cfDNA) (Thermo Fisher Scientific, Waltham, Massachusetts), ${ }^{4}$ and citrullinated histone $\mathrm{H} 3$ (Cayman chemicals, Ann Arbor, Michigan) were determined using commercially available reagents, according to the manufacturers' protocols.

Statistical analysis: A 60-day mortality and exacerbationfree survival rate were determined. Exacerbation was defined as the disease recurred within 30 days after the cessation of 
TPE. All statistical analyses were performed with the SPSS 26.0 software. For data with normal distribution, values were expressed as the mean \pm standard deviation. For data that is not normally distributed, the values were expressed as the median and interquartile range. Student $t$-test was used to determine differences between two groups with normally distributed data, while the Mann-Whitney U test was used to analyze data that were not normally distributed. Log-rank test was used to compare the death-free survival rate between two groups. $p$-Values of less than 0.05 and less than 0.01 were considered statistically significant and highly statistically different, respectively.

\section{Results}

Patient characteristics: Of 109 episodes with a confirmed diagnosis of iTTP(i.e., ADAMTS13 activity less than $10 \mathrm{IU} / \mathrm{dL}$ or $10 \%$ of normal, and inhibitor $>0.4 \mathrm{U} / \mathrm{mL}$ or anti-ADAMTS13 IgG $>15$ $\mathrm{U} / \mathrm{mL}), 66$ (60.5\%) presented with neurological signs and symptoms. Review of the patients' electronic medical record found that $14(21.2 \%)$ of these patients with neurological complaints did not have neuroimaging study (CT/MRI) done during the entire hospitalization. However, 52 (78.8\%) patients did have CT/MRI done, which was available for imaging review. Of these, 22 (33\%) patients had confirmed stroke on CT/MRI, including both small and large vessels strokes, and $30(45.4 \%)$ patients had no evidence of stroke on CT/MRI. For further analysis, the patients without any imaging and those with no evidence of stroke were combined into one group as "without stroke."

Association of patient demographics and comorbidities with stroke: Of 66 patients presented with neurological signs and symptoms, the mean age was 45.2 years (standard deviation, SD of 13.7 years) at the time of presentation. The patients with imaging-confirmed stroke were older (the mean age, 48.7 years) as compared with those without stroke (the mean age, 43.4 years). There was no difference in gender as shown in -Table 1. However, of those with imaging-confirmed stroke, $13 / 22$ (59.1\%) were females. Of 66 patients in the study cohort, 49 (74.2\%) were African American (AA), and of those 15
(68.2\%) had imaging-confirmed stroke, significantly higher than that in all iTTP patients.

Only 14 (21.2\%) patients were presenting as initial episode, and remainder were admitted as a relapse. Majority of the patients had one or more associated comorbidities, 40 (60.6\%) had an associated hypertension $(p=0.02)$. History of smoking was available in 56 patients. Of those, 27 (48.2\%) of the patients had history of smoking or were current smokers. The association was statistically significant in patients with imaging confirmed stroke $(p=0.021)$. Only $16(24.2 \%)$ of patients with neurological symptoms had diabetes mellitus and it was not statistically significant (-Table $\mathbf{1}$ ).

Correlation of laboratory parameters in patients with stroke: The routine laboratory parameters were consistent with the diagnosis of iTTP in all patients as shown in -Table 2. The mean platelet count in the patients was $12.8 \times 10^{9} / \mathrm{L}$, with no significant difference in between groups with and without imaging-confirmed stroke. The mean hemoglobin and hematocrit were 8.4 and $24.4 \%$, respectively. PT, activated thromboplastin time, and serum fibrinogen were in normal range (-Table 2 ). Serum D-dimer was elevated in all patients with the mean value of $1,902.5 \mathrm{ng} / \mathrm{mL}$, which was significantly increased in patients with imaging-confirmed stroke $(p=0.046)$. All patients included for this cohort had confirmed diagnosis of iTTP with ADAMTS13 activity less than $10 \mathrm{U} / \mathrm{dL}$ or $10 \%$ of normal. ADAMTS13 inhibitors were available for only 36 patients with the mean value of $1.4 \mathrm{BU}$ and there was no statistically significant difference between the two groups (-Table $\mathbf{3}$ ). Additionally, plasma levels of anti-ADAMTS13IgG were all above the normal range. The levels were significantly higher in patients with than those without an imaging-confirmed stroke $(p=0.003)$ ( - Fig. 1 ; - Table 3 ).

To assess if acute inflammation, neutrophil activation, and NETosis play a role in pathogenesis of stroke, we determined plasma NETosis markers including histone/DNA complexes, citrH3, and cfDNA in these patients. To our surprise, while plasma levels of histone/DNA complexes, citH3, and cfDNA were dramatically elevated in all patients with acute iTTP,

Table 1 Clinical characteristics in all iTTP patients with or without an imaging-confirmed ischemic stroke

\begin{tabular}{|l|l|l|l|l|}
\hline Clinical information & Total $N=66$ & Without stroke $(N=44)$ & Confirmed stroke $(N=22)$ & $p$-Value \\
\hline Age (year) & $45.2 \pm 13.7$ & $43.4 \pm 14.1$ & $48.7 \pm 12.3$ & 0.142 \\
\hline Female, $n(\%)$ & $33(50)$ & $20(45.5)$ & $13(59.1)$ & 0.434 \\
\hline African American, $n(\%)$ & $49(74.2)$ & $34(77.3)$ & $15(68.2)$ & 0.309 \\
\hline Blood group O, $n(\%)$ & $41(62.1)$ & $29(65.9)$ & $12(54.5)$ & 0.426 \\
\hline Initial episode, $n(\%)$ & $14(21.2)$ & $6(13.6)$ & $8(36.4)$ & 0.054 \\
\hline Hypertension, $n(\%)$ & $40(60.6)$ & $22(50)$ & $18(81.8)$ & $0.027^{\text {a }}$ \\
\hline Diabetes mellitus, $n(\%)$ & $16(24.2)$ & $13(29.5)$ & $3(13.6)$ & 0.226 \\
\hline Smoking $(N=56), n(\%)$ & $27(48.2)$ & $14(36.8)$ & $13(72.2)$ & $0.021^{\text {a }}$ \\
\hline SLE $(N=38), n(\%)$ & $3(7.9)$ & $3(13.6)$ & 0 & \\
\hline
\end{tabular}

Abbreviations: iTTP, immune thrombotic thrombocytopenic purpura; $N$, total cases evaluated; $n$, the number of cases in each subgroup; SLE, systemic lupus erythematatosus.

${ }^{a} p<0.05$ indicates the statistical significance. 
Table 2 Routine laboratory parameters in iTTP patients with or without imaging-confirmed ischemic stroke

\begin{tabular}{|c|c|c|c|c|}
\hline Laboratory parameters & All $(N=66)$ & $\begin{array}{l}\text { Without stroke } \\
(N=44)\end{array}$ & $\begin{array}{l}\text { Confirmed stroke } \\
(N=22)\end{array}$ & p-Value \\
\hline WBC $\left(\times 10^{9} / \mathrm{L}\right)$, mean \pm SD & $10.8(7.6-13.2)$ & $11.1(7.8-13.2)$ & $8.7(7.6-12.7)$ & 0.297 \\
\hline $\mathrm{Hb}(\mathrm{g} / \mathrm{dL})$, mean $\pm \mathrm{SD}$ & $8.4 \pm 1.7$ & $8.4 \pm 1.6$ & $8.5 \pm 1.9$ & 0.81 \\
\hline Hct $(\%)$, mean $\pm S D$ & $24.4 \pm 4.9$ & $24.2 \pm 4.7$ & $24.3 \pm 5.4$ & 0.641 \\
\hline Platelet count $\left(\times 10^{9} / \mathrm{L}\right)$, median (IQR) & $12.8(8.6-19.4)$ & $14(8.4-19.4)$ & $12(8.6-24.2)$ & 0.948 \\
\hline Creatinine (mg/dL), median (IQR), $n=63$ & $1.3(0.9-1.7)$ & $1.3(1.0-1.6)$ & $1.3(0.9-1.8)$ & 0.726 \\
\hline PT (s), median (IQR), $n=61$ & $14.9(14.1-15.9)$ & $14.9(14.3-15.7)$ & $14.9(14-16.1)$ & 0.92 \\
\hline $\operatorname{aPPT}(s)$, mean $\pm S D, n=58$ & $31.7 \pm 5.8$ & $31.3 \pm 508$ & $32.4 \pm 6.0$ & 0.515 \\
\hline Fibrinogen $(\mathrm{mg} / \mathrm{dL}), n=55$, mean $\pm \mathrm{SD}$ & $401.3 \pm 131.9$ & $385.5 \pm 139.4$ & $431.3 \pm 113.8$ & 0.224 \\
\hline D-dimer (ng/dL), median (IQR), $n=63$ & $\begin{array}{l}1,902.5 \\
(1,086.3-5,195.8)\end{array}$ & $\begin{array}{l}2,631 \\
(1,370-5,804) \\
\end{array}$ & $\begin{array}{l}1,138 \\
(715.5-2,498)\end{array}$ & $0.046^{\mathrm{a}}$ \\
\hline Troponin-I (ng/dL), median (IQR) & $0.5(0.1-1.3)$ & $0.5(0.1-1.2)$ & $0.7(0.2-2.8)$ & 0.273 \\
\hline
\end{tabular}

Abbreviations: aPTT, activated thromboplastin time; Hct, hematocrit; IQR, interquartile range; iTTP, immune thrombotic thrombocytopenic purpura; $N$, total number of cases; $n$, number of cases in this category; PT, prothrombin time; WBC, white blood cells.

${ }^{a} p$-Value $<0.05$ indicates statistical significance.

Table 3 Special laboratory parameters in iTTP patients with or without imaging-confirmed ischemic stroke

\begin{tabular}{|c|c|c|c|c|}
\hline & All cases $(N=66)$ & $\begin{array}{l}\text { Without stroke } \\
(N=44)\end{array}$ & $\begin{array}{l}\text { Confirmed stroke } \\
(N=22)\end{array}$ & $p$-Value \\
\hline $\begin{array}{l}\text { Anti-ADAMTS13 IgG } \\
(\mathrm{U} / \mathrm{mL}), n=36\end{array}$ & $5,022(1,954-7,929)$ & $3,307(1,588-5,486)$ & $7,634(5,638-1,2830)$ & $0.003^{a}$ \\
\hline Inhibitor (U/mL), $n=64$ & $1.4(0.5-4.6)$ & $1.4(0.5-4.0)$ & $1.4(0.5-5.6)$ & 0.92 \\
\hline CitH3 (ng/mL), $n=45)$ & $4.4(2.5-7.0)$ & $4.9(2.1-7.2)$ & $3.5(2.6-7.1)$ & 0.758 \\
\hline $\begin{array}{l}\text { Histone/DNA complexes } \\
(\mathrm{U} / \mathrm{mL}), n=42)\end{array}$ & $69.3(37.1-144.4)$ & $69.2(38.0-142.2)$ & $80.6(33.6-160.4)$ & 0.703 \\
\hline cfDNA (ng/mL), $n=48)$ & $983(812-1,286)$ & $1,040(824-1,701)$ & $913(791-1,122)$ & 0.616 \\
\hline
\end{tabular}

Abbreviations: $N$, total number of cases; $n$, number of cases assayed for this biomarker.

${ }^{a} p$-Value $<0.01$ indicates that the difference between two groups is statistically highly significant.

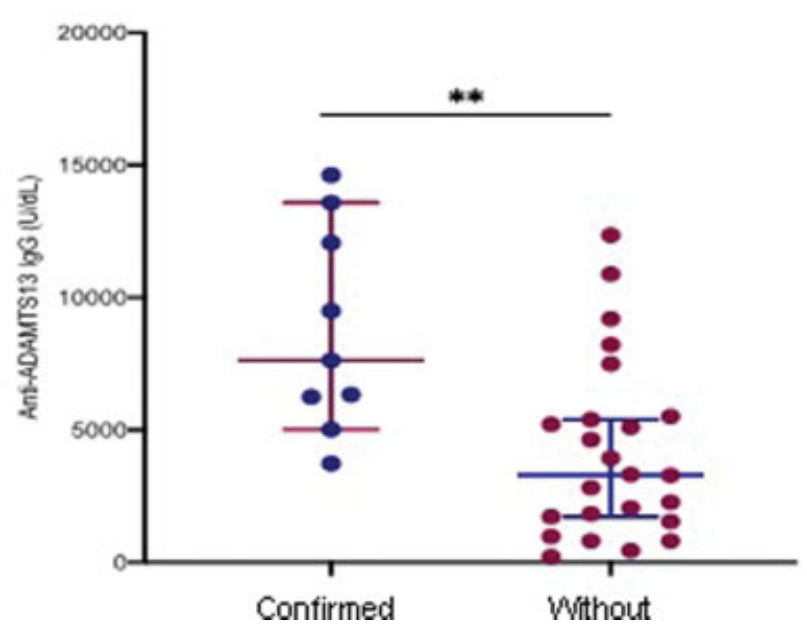

Fig. 1 Plasma anti-ADAMTS13 IgG in patients with iTTP with (A) or without (B) imaging-confirmed stroke. Each dot represents each patient value; the horizontal bars inside dots are median and interquartile range (IQR). Mann-Whitney test was performed for statistical significance between two groups. $p<0.05$ is statistically significant. their levels in iTTP patients with stroke were not significantly higher than those without stroke (-Table 3).

Association of stroke with mortality and exacerbation: The 60-day mortality and exacerbation-free survival were determined in all patients. Using the log-rank test, we found no association of 60-day mortality and exacerbation-free survival rate with stroke. As shown in -Fig. 2, Kaplan-Meier survival analysis demonstrated that there was no statistically significant difference in 60-day mortality rate and exacerbation-free survival rate between patients with and without imaging-confirmed stroke, suggesting that ischemic stroke does not adversely affect patient's survival or predicts exacerbation during acute episode while the long-term impact on the brain function is not known.

\section{Discussion}

In the present study, we report the clinical and laboratory characteristics of 66 patients with acute iTTP who presented with neurological signs and symptoms in a single institution. 

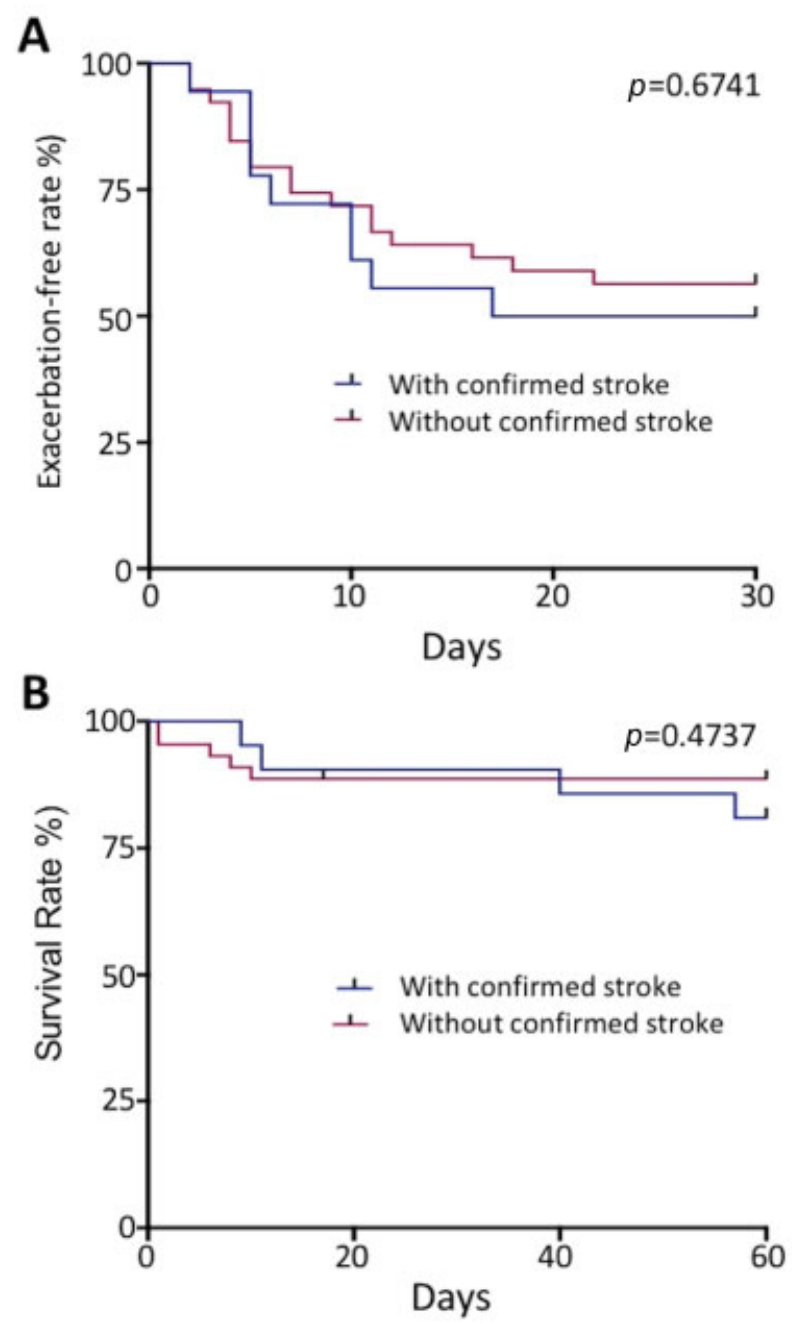

Fig. 2 Kaplan-Meier survival analysis in patients with or without stroke. (A) Exacerbation free rate (\%) in patients with or without stroke 30 days following the cessation of TPE; (B) 60-day survival rate in patients with or without stroke since admission.

These patients are divided into two groups, based on the presence or the absence of cerebral infarction on the neuroimaging study. We found that older age, black race, and comorbidities such as cardiovascular disease, diabetes, and smoking, are associated with ischemic cerebral infarction. So were plasma levels of anti-ADAMTS13 IgG, but not the inflammatory or NETosis markers, 60-day mortality, and exacerbation.

Most published studies to date are either a case report or small series of report that addressed the stroke in patients with acute TTP. ${ }^{5-7}$ Burrus et al reported 46 patients with TTP who had neuroimaging done. ${ }^{24}$ No association of imagingconfirmed stroke with comorbidities was found. In our study, more than half of the iTTP patients presented with one or more neurological sign or symptoms as their initial complaint on admission. The symptoms range from headache, dizziness, numbness and weakness in the extremities, confusion to loss of consciousness, visual changes, disorientation, altered mental status, and generalized seizures. In our cohort, no patient presented with headache as the sole neurological symptom. Surprisingly, in two-third cases in which neuroimaging was performed approximately $42.5 \%$ had imaging-confirmed small and large vessel stroke. There was no significant difference in gender of patients presenting with neurological symptoms. However, there was female predominance in patient with imaging-confirmed stroke. Patients with imaging confirmed stroke were older than those without stroke. As previously reported, majority of our such patients were African Americans, ${ }^{4,12}$ and also majority of the patients with neurological signs and symptoms were presenting with a relapse disease or sometime during remission (when platelet count was normal). Black is shown to be at risk for the development of autoantibody against ADAMTS13 and mortality, ${ }^{39}$ resulting from lack of the protective HLA-DRB $1{ }^{*} 04 .{ }^{40}$

Analyzing other risk factors in these patients, all patients with imaging-confirmed stroke showed a significant association with hypertension and smoking. This may not be the surprising findings as the association of hypertension with vascular damage and thromboembolic events in TTP patients has been well established. ${ }^{41,42}$ However, the association with smoking in iTTP patients with stroke has not been previously observed. In view of the clinical manifestations of the iTTP that often involve serious consequences, such as thrombotic events, it is necessary to know other factors and conditions that may result in a worse outcome. ${ }^{43,44}$ There was no significant association of iTTP stroke with diabetes in our study.

The D-dimer was significantly increased in all patients diagnosed with acute iTTP, which was strongly correlated with an imaging-confirmed stroke. Consistent with those previously reported, the high levels of anti-ADAMTS13 IgG correlate with disease progression and potential relapse in patients with acute iTTP. ${ }^{38,45}$ However, the strong association between anti-ADAMTS13 IgG levels (but not inhibitor titer) and stroke had not previously observed. To our surprise, there was no association between plasma levels of histone/DNA complexes, citH3, and cfDNA and imaging confirmed stroke, despite a marked elevation of these markers in patients with acute iTTP compared with the healthy controls or in patients during remission. $4,46,47,49$ These inflammatory and NETosis markers appear to be associated with the size of ischemic stroke $^{48}$ and in-hospital mortality. ${ }^{49}$

We recognize the potential limitations in the present study. First, not all iTTP patients had neuroimaging done on admission and during hospitalization; second, some of the clinical and imaging information were obtained retrospectively, which may not be complete; third, the number of cases in both groups were relatively small due to the rarity of iTTP. This may have a low statistical power to detect the difference in exacerbation or mortality rate in patients with or without stroke.

In conclusion, our present study demonstrates a proof of concept that patients with acute iTTP who present with neurological signs and symptoms may have a higher incidence of ischemic stroke than we have previously known. The risk factors for developing such an ischemic stroke in iTTP patients include older age, comorbid hypertension, and smoking; additionally, high admission plasma levels of anti-ADAMTS13 
IgG, but not inflammatory/NETosis markers, are associated with ischemic stroke. To our surprise, no correlation between the ischemic stroke and iTTP recurrence or mortality was identified, although the long-term neurological sequelae of stroke were yet to be assessed. We propose that a prospective and longitudinal neuroimaging study for patients with iTTP should be conducted to assess the incidence rate, risk factors, reversibility, and long-term outcomes associated with ischemic stroke.

\section{Authors' Contributions}

R.M., C.L., J.S., and X.L.Z. designed research, performed experiments, and analyzed the results, as well as wrote the manuscript. All other nonauthors in the acknowledgment contribute to patient recruitment, informed consents, and sample collections. All authors have reviewed and approved the final version of the manuscript for submission.

\section{Funding}

The study is supported in part by grants from NHLBI (HL126724 to XLZ).

\section{Conflict of Interest}

X.L.Z. is a speaker or consultant for Alexion, SanofiGenzyme, and Takeda. X.L.Z. is also the Founder of Clotsolution. All other authors have declared no relevant conflict.

\section{Acknowledgments}

The authors appreciate all pathology attendings, residents, fellows, and apheresis nurses, medical technologists in the coagulation laboratories for their assistance in informed consent, sample collection, and sample preparation, and storage.

\section{References}

1 Moake JL. Thrombotic thrombocytopenic purpura: the systemic clumping "plague". Annu Rev Med 2002;53:75-88

2 Zheng XL, Sadler JE. Pathogenesis of thrombotic microangiopathies. Annu Rev Pathol 2008;3:249-277

3 Terrell DR, Williams LA, Vesely SK, Lämmle B, Hovinga JA, George JN. The incidence of thrombotic thrombocytopenic purpura-hemolytic uremic syndrome: all patients, idiopathic patients, and patients with severe ADAMTS-13 deficiency.J Thromb Haemost 2005;3(07): 1432-1436

4 Staley EM, Cao W, Pham HP, et al. Clinical factors and biomarkers predict outcome in patients with immune-mediated thrombotic thrombocytopenic purpura. Haematologica 2019;104(01):166-175

5 Rinkel GJ, Wijdicks EF, Hené RJ. Stroke in relapsing thrombotic thrombocytopenic purpura. Stroke 1991;22(08):1087-1089

6 Downes KA, Yomtovian R, Tsai HM, Silver B, Rutherford C, Sarode R. Relapsed thrombotic thrombocytopenic purpura presenting as an acute cerebrovascular accident. J Clin Apher 2004;19(02):86-89

7 Boattini M, Procaccianti G. Stroke due to typical thrombotic thrombocytopenic purpura treated successfully with intravenous thrombolysis and therapeutic plasma exchange. BMJ Case Rep 2013;2013:bcr2012008426

8 Wiernek SL, Jiang B, Gustafson GM, Dai X. Cardiac implications of thrombotic thrombocytopenic purpura. World J Cardiol 2018;10 (12):254-266
9 Benhamou Y, Boelle PY, Baudin B, et al;Reference Center for Thrombotic Microangiopathies Experience of the French Thrombotic Microangiopathies Reference Center. Cardiac troponin-I on diagnosis predicts early death and refractoriness in acquired thrombotic thrombocytopenic purpura. J Thromb Haemost 2015;13(02):293-302

10 Atreya AR, Arora S, Sivalingam SK, Giugliano GR. ST segment elevation myocardial infarction as a presenting feature of thrombotic thrombocytopenic purpura. J Cardiovasc Dis Res 2012;3 (02):167-169

11 Bell WR, Braine HG, Ness PM, Kickler TS. Improved survival in thrombotic thrombocytopenic purpura-hemolytic uremic syndrome. Clinical experience in 108 patients. N Engl J Med 1991;325 (06):398-403

12 Zheng XL, Kaufman RM, Goodnough LT, Sadler JE. Effect of plasma exchange on plasma ADAMTS13 metalloprotease activity, inhibitor level, and clinical outcome in patients with idiopathic and nonidiopathic thrombotic thrombocytopenic purpura. Blood 2004;103(11):4043-4049

13 Page EE, Kremer Hovinga JA, Terrell DR, Vesely SK, George JN. Thrombotic thrombocytopenic purpura: diagnostic criteria, clinical features, and long-term outcomes from 1995 through 2015. Blood Adv 2017;1(10):590-600

14 Nunez Zuno JA, Khaddour K. Thrombotic Thrombocytopenic Purpura Evaluation and Management. In: StatPearls [Internet]. Treasure Island, FL: StatPearls Publishing; 2020

15 Scully M, Cataland S, Coppo P, et al;International Working Group for Thrombotic Thrombocytopenic Purpura. Consensus on the standardization of terminology in thrombotic thrombocytopenic purpura and related thrombotic microangiopathies. J Thromb Haemost 2017;15(02):312-322

16 Scully M, Hunt BJ, Benjamin S, et al;British Committee for Standards in Haematology. Guidelines on the diagnosis and management of thrombotic thrombocytopenic purpura and other thrombotic microangiopathies. Br J Haematol 2012;158(03): 323-335

17 Saha M, McDaniel JK, Zheng XL. Thrombotic thrombocytopenic purpura: pathogenesis, diagnosis and potential novel therapeutics. J Thromb Haemost 2017;15(10):1889-1900

18 Zheng XL, Vesely SK, Cataland SR, et al. ISTH guidelines for the diagnosis of thrombotic thrombocytopenic purpura. J Thromb Haemost 2020;18(10):2486-2495

19 Zheng XL. ADAMTS13 and von Willebrand factor in thrombotic thrombocytopenic purpura. Annu Rev Med 2015;66:211-225

20 Lämmle B, Kremer Hovinga JA, Alberio L. Thrombotic thrombocytopenic purpura. J Thromb Haemost 2005;3(08):1663-1675

21 Furlan M, Robles R, Lämmle B. Partial purification and characterization of a protease from human plasma cleaving von Willebrand factor to fragments produced by in vivo proteolysis. Blood 1996; 87(10):4223-4234

22 Tsai HM, Lian EC. Antibodies to von Willebrand factor-cleaving protease in acute thrombotic thrombocytopenic purpura. N Engl J Med 1998;339(22):1585-1594

23 Rock GA, Shumak KH, Buskard NA, et al;Canadian Apheresis Study Group. Comparison of plasma exchange with plasma infusion in the treatment of thrombotic thrombocytopenic purpura. N Engl J Med 1991;325(06):393-397

24 Burrus TM, Wijdicks EF, Rabinstein AA. Brain lesions are most often reversible in acute thrombotic thrombocytopenic purpura. Neurology 2009;73(01):66-70

25 Fugate JE, Claassen DO, Cloft HJ, Kallmes DF, Kozak OS, Rabinstein AA. Posterior reversible encephalopathy syndrome: associated clinical and radiologic findings. Mayo Clin Proc 2010;85(05):427-432

26 Rock G. Plasma exchange in the management of thrombotic thrombocytopenic purpura. Vox Sang 2002;83(Suppl 1):141-143

27 Peyvandi F, Scully M, Kremer Hovinga JA, et al;TITAN Investigators. Caplacizumab for acquired thrombotic thrombocytopenic purpura. N Engl J Med 2016;374(06):511-522 
28 Scully M, Cataland SR, Peyvandi F, et al;HERCULES Investigators. Caplacizumab treatment for acquired thrombotic thrombocytopenic purpura. N Engl J Med 2019;380(04):335-346

29 Zheng XL, Vesely SK, Cataland SR, et al. ISTH guidelines for treatment of thrombotic thrombocytopenic purpura. J Thromb Haemost 2020;18(10):2496-2502

30 Jestin M, Benhamou Y, Schelpe AS, et al;French Thrombotic Microangiopathies Reference Center. Preemptive rituximab prevents long-term relapses in immune-mediated thrombotic thrombocytopenic purpura. Blood 2018;132(20):2143-2153

31 Westwood JP, Thomas M, Alwan F, et al. Rituximab prophylaxis to prevent thrombotic thrombocytopenic purpura relapse: outcome and evaluation of dosing regimens. Blood Adv 2017;1(15):1159-1166

32 Westwood JP, Webster H, McGuckin S, McDonald V, Machin SJ, Scully M. Rituximab for thrombotic thrombocytopenic purpura: benefit of early administration during acute episodes and use of prophylaxis to prevent relapse. J Thromb Haemost 2013;11(03): 481-490

33 Froissart A, Buffet M, Veyradier A, et al; French Thrombotic Microangiopathies Reference Center Experience of the French Thrombotic Microangiopathies Reference Center. Efficacy and safety of first-line rituximab in severe, acquired thrombotic thrombocytopenic purpura with a suboptimal response to plasma exchange. Crit Care Med 2012;40(01):104-111

34 Schleinitz N, Ebbo M, Mazodier K, et al. Rituximab as preventive therapy of a clinical relapse in TTP with ADAMTS13 inhibitor. Am J Hematol 2007;82(05):417-418

35 Bresin E, Gastoldi S, Daina E, et al. Rituximab as pre-emptive treatment in patients with thrombotic thrombocytopenic purpura and evidence of anti-ADAMTS13 autoantibodies. Thromb Haemost 2009;101(02):233-238

36 Scully M, McDonald V, Cavenagh J, et al. A phase 2 study of the safety and efficacy of rituximab with plasma exchange in acute acquired thrombotic thrombocytopenic purpura. Blood 2011;118 (07):1746-1753

37 Montoya RC, Poiesz BJ. Rituximab as prophylaxis in chronic relapsing thrombotic thrombocytopenic purpura: a case report and review of the literature. Blood Coagul Fibrinolysis 2012;23 (04):338-341

38 Sui J, Cao W, Halkidis K, et al. Longitudinal assessments of plasma ADAMTS13 biomarkers predict recurrence of immune thrombotic thrombocytopenic purpura. Blood Adv 2019;3(24):4177-4186
39 Martino S, Jamme M, Deligny C, et al;French Reference Center for Thrombotic Microangiopathies. Thrombotic thrombocytopenic purpura in Black People: impact of ethnicity on survival and genetic risk factors. PLoS One 2016;11(07):e0156679

40 Terrell DR, Motto DG, Kremer Hovinga JA, Lämmle B, George JN, Vesely SK. Blood group O and black race are independent risk factors for thrombotic thrombocytopenic purpura associated with severe ADAMTS13 deficiency. Transfusion 2011;51(10):2237-2243

41 van den Born BJ, van der Hoeven NV, Groot E, et al. Association between thrombotic microangiopathy and reduced ADAMTS13 activity in malignant hypertension. Hypertension 2008;51(04):862-866

42 van den Born BJ, Honnebier UP, Koopmans RP, van Montfrans GA. Microangiopathic hemolysis and renal failure in malignant hypertension. Hypertension 2005;45(02):246-251

43 Chaturvedi S, Carcioppolo D, Zhang L, McCrae KR. Management and outcomes for patients with TTP: analysis of 100 cases at a single institution. Am J Hematol 2013;88(07):560-565

44 Chaturvedi S, Bhatia N. Predictors of survival in thrombotic thrombocytopenic purpura. Haematologica 2013;98(05):e58

45 FerrariS, Scheiflinger F, Rieger M, et al;French Clinical and Biological Network on Adult Thrombotic Microangiopathies. Prognostic value of anti-ADAMTS 13 antibody features (Ig isotype, titer, and inhibitory effect) in a cohort of 35 adult French patients undergoing a first episode of thrombotic microangiopathy with undetectable ADAMTS 13 activity. Blood 2007;109(07):2815-2822

46 Cao WJ, Pham HP, Williams LA, et al. Increased plasma levels of human neutrophil peptides (HNPs) and complement activation markers in patients with acquired thrombotic thrombocytopenic purpura (TTP). Blood 2015;126:1147

47 Fuchs TA, Kremer Hovinga JA, Schatzberg D, Wagner DD, Lämmle B. Circulating DNA and myeloperoxidase indicate disease activity in patients with thrombotic microangiopathies. Blood 2012;120 (06):1157-1164

48 Lin C, Memon RA, Sui J, Zheng XL. Identification of biomarkers in patients with thrombotic thrombocytopenic purpura presenting with large and small ischemic strokes. Cerebrovas Dis EXTRA 2020. In press

49 Sui J, Lu RN, Halkidis K, et al. Plasma levels of S100A8/A9, histone/DNA complexes, and cell-free DNA are associated with mortality in patients with immune thrombotic thrombocytopenic purpura. Thromb Haemost 2020 (e-pub ahead of print). Doi: $10.1111 /$ jth.15176 